\title{
Modeling and Analysis of Integrated Spiral Inductors for RF System-in-Package
}

\author{
Minqing Liu \\ Computer Engineering \\ University of California, Santa Cruz \\ Santa Cruz,CA 95064 \\ Tel: 831-459-4954 \\ Fax: 831-459-4954 \\ e-mail: liumq@cse.ucsc.edu
}

\author{
Wayne W.-M. Dai \\ Computer Engineering \\ University of California, Santa Cruz \\ Santa Cruz, CA 95064 \\ Tel: $831-459-4234$ \\ Fax: 831-459-4829 \\ e-mail dai@cse.ucsc.edu
}

\begin{abstract}
In this paper, a fast efficient Method of Moments(MoM) based on a new subdomain partitioning technique is presented for rapidly extracting the distributed capacitance and inductance of spiral inductors. By using this approach, the analytical formula of Green's function for multi-layer substrates is obtained through the prioritized wavetracing method. The computing time is much faster than the state of art capacitance and inductance solvers, such as FMMS[6]. Good agreement between numerical results and measurement data is shown to demonstrate the accuracy of the proposed new method.
\end{abstract}

\section{INTRODUCTION}

Communication is becoming more and more intelligent and mobile. TV, pager, cellular, GPS, and wireless modem are all working in the frequency range of $500 \mathrm{MHz}-$ $5 \mathrm{GHz}$. Cellular and GPS are enjoying a huge market in the world. This huge emerging wireless market demands "single-module" or "single-chip" solutions for radio frequency integrated circuits. The relative merits of integrated circuits over conventional hybrid circuits in microwave system design are in terms of cost, size, weight, high-volume production capability, design flexibility, broad-band performance, high level of integration, reproducibility and reliability. However, compared with other analog circuits, RF wireless applications generally make more extensive use of reactive passive components, especially inductors. Many of the passive components are poor candidates for on-chip integration due to their large values and stringent requirements on their high quality factor, Q. Integrated passive components on silicon substrates together with highly integrated CMOS die in a single package, which is one example of system-in-package (SIP), provides new opportunities to achieve the hybridcircuit functionalities in a single integrated module. The SIP technology not only provides an interconnect mechanism for both digital and RF circuits, but also includes a unique feature of building integrated passive components.

The advantage of placing inductors on the substrate instead of on chip is that with the high resistivity substrate and wide/thick metal lines, we can achieve significantly high $\mathrm{Q}$, which is desired in the RF wireless design, in an inexpensive process. Furthermore, by integrating the passive components inside the package, we can significantly reduce undesirable parasitics and improve the predictability of circuit behavior, while still keep the advantage of system-on-chip solution, such as small size, low power, and high performance.

Fig.1 illustrates the cross section of a typical SIP technology for RF applications and Fig.2 shows one implementation of a typical SIP. The advantages of SIP cannot be realized without rigorous modeling prior to circuit design. Among these integrated passive components, the integrated inductor is the most difficult to be properly characterized and designed as in silicon bipolar process. The most challenge job is to predict the coupling effects between inductors and other devices in relative large structure. However traditional full-wave solvers limit their uses because they are computation prohibited. Therefore, the RF design of wireless SIP calls for accurate and efficient modeling and analysis of integrated passive components.

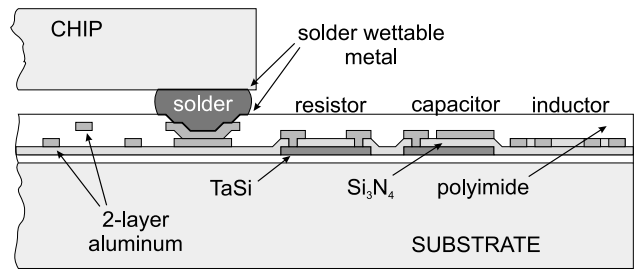

Fig. 1. The cross section of a typical SIP technology. 


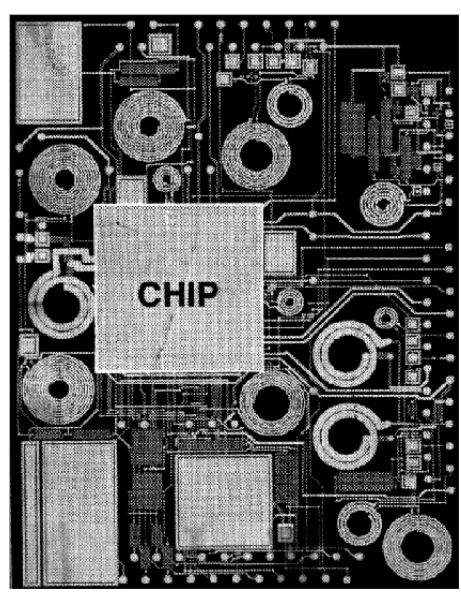

Fig. 2. An implementation of typical SIP technology with many spiral inductors.

In the early time before the numerical methods being developed, the analytic methods usually based on $\mathrm{H}$. M. Greenhouse methods[1, 2] or coupled microstrip line theory[3]. While the H. M. Greenhouse methods have low accuracy, methods based on coupled microstrip line theory have geometric limitation. Arun Balakrishnan[11] presented two methods for calculating the inductance of planar spiral inductors. The first method assumes current flows only in the exact center of the conductor. The second method is based on uniform current distribution across the conductor width, and results in some improvements in the calculated value of inductance. But it still limited by the coupled microstrip line theory. To achieve the first pass success of manufacturing integrated spiral inductors in SIP substrate, high accuracy and robustness in inductor modeling and analysis are critical. The numerical methods, such as Method of Moments (MoM), Finite Element, were applied to the inductor modeling. Among them MoM is known as a more accurate one. MoM relies on integral equation formulations which have several advantages such as good conditioning, the ability to treat arbitrary regions, but have one overriding disadvantage: the high cost of working with large dense matrices. In the last decade, a number of algorithms[7, 8, 9] have been developed for the fast solution of dense matrices arising from integral equation formulations. Recently the singular value decomposition (SVD) method[5] has been used to accelerate the computation of integral equation. The algorithm adaptively partitions the matrix into low rank sub-matrices via a divide and conquer scheme. By using local SVDs, the low-rank sub-matrices are compressed. Although the new SVD-accelerated solver significantly reduce the computing time, it is still too slow to perform coupling analysis and design optimization. In this paper, we propose a new technique to calculate the
Green's function. Our technique takes advantages of the sub-domain partitioning for MoM. With sub-domain partitioning, Green's function can be deduced analytically. By using this analytical formula, we can obtain Green's function for multi-layered substrates through the prioritized wave-tracing method, which takes only a fraction of time needed by other methods. While others partitioned the inductor into subdomain of triangle geometric forms, which resulted in a large dense matrix, we divide subdomain along the circular loop. This produces a small matrix, therefore significantly reduces the computing time. For example the parameter extraction of the 3 -turn spiral inductor take only few seconds.

\section{Calculation of Inductance}

The configuration of a circular spiral inductor and its equivalent circuit are shown in Fig.3. The circular spiral inductor is modeled through the following principles: the geometric formation of spiral is approximately axisymmetric; The $N$-turn inductor can be approaximated as $N$-turn circular microstrip line with the same center. Suppose a spiral inductor has $N$ turn loops, denoted as $1^{s t}, 2^{n d}, \cdots, N^{t h}$ turn, with the common loop width $W$. Every loop is further divided into $M$ turns of sub-loops, with sub-loops width $W / M$ as illustrated in Fig.4. In the spiral structure shown in Fig.3, the outermost turn is of dimension $d$, and adjacent inside turns all have spacing $s$. The spiral structure may be approximated by a system of concentric turns, all having the same center-to-center spacing $s$ as before. The diameter of $j^{t h}$ turn, $j$ counted from the outside to the inside, is $d-2(j-1)(s+w)$.

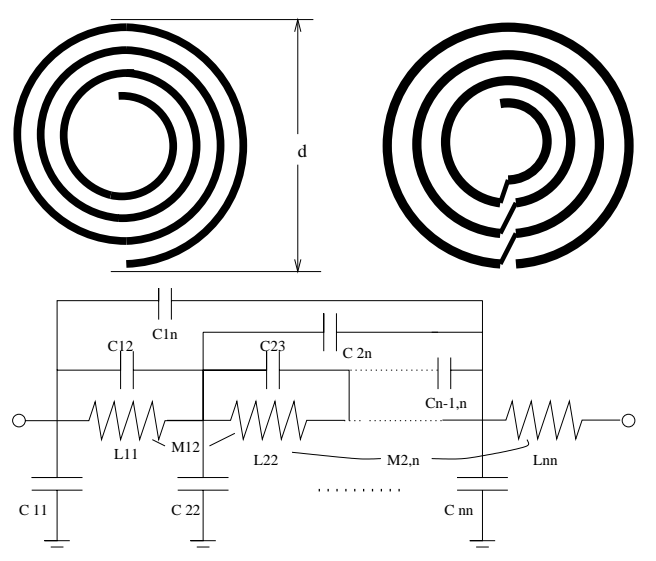

Fig. 3. Physical structure of the inductor and equivalent circuit.

The total inductance of planar spiral inductor with $N$ loops is 


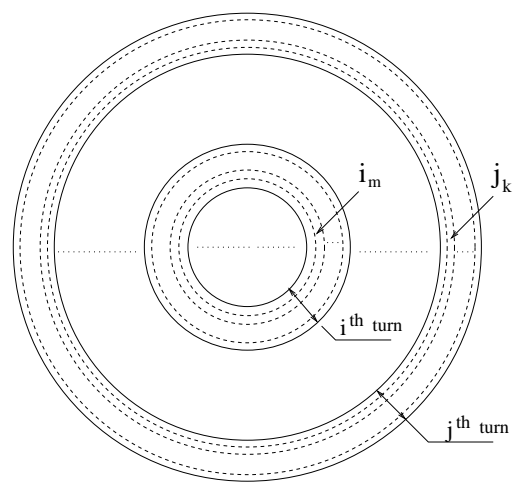

Fig. 4. Each loop is divided into $M$ turns of sub-loop.

$$
L=\sum_{i=1}^{N} \sum_{j=1}^{N} M_{i j}
$$

where $M_{i j}$, the mutual inductance between $i^{t h}$ and $j^{t h}$ turn, can be obtained by

$$
M_{i j}=\sum_{k=1}^{M} \sum_{m=1}^{M} M_{i_{k}, j_{m}}
$$

$i_{k}^{t h}$ and $j_{m}^{t h}$ denote the $k^{t h}$ sub-loop in $i^{t h}$ turn and the $m^{t h}$ sub-loop in the $j^{\text {th }}$ turn respectively. The self-inductance of $i^{t h}$ turn $L_{i}$ can be derived from Eq.2 by letting $i=j$. $M_{i_{k}, j_{m}}$, the mutual inductance between the $k^{t h}$ sub-loop in $i^{t h}$ turn and the $m^{t h}$ sub-loop in the $j^{\text {th }}$ turn, can be obtained from

$$
M_{i_{k}, j_{m}}=\frac{1}{I_{j_{m}}} \oint_{l_{i_{k}}} \bar{A} \cdot \overline{d l}_{i_{k}}
$$

where the magnetic vector potential produced by the circular current flowing in $j_{m}^{t h}$ sub-loop:

$$
\bar{A}=\mu_{0} \oint_{l_{j_{m}}} G\left(\rho, \rho^{\prime}\right) \cdot J_{j_{m}} \cdot \overline{d l}_{j_{m}}
$$

$J_{i_{m}}$ and $J_{j_{k}}$ are current densities on the $i_{m}^{t h}$ sub-loop and $j_{k}^{t h}$ sub-loop, respectively. Combinning Eq.2, Eq.3, and Eq.4, we have the mutual inductance between the $i^{\text {th }}$ and $j^{\text {th }}$ turn

$$
M_{i j}=\frac{\mu_{0} \sum_{m=1}^{N} \sum_{k=1}^{N} J_{i_{k}} J_{j_{m}} \oint_{l_{i_{k}}} \oint_{l_{j_{m}}} G\left(\rho, \rho^{\prime}\right) \cdot \overline{d l}_{i_{k}} \overline{d l}_{j_{m}}}{\sum_{k=1}^{N} J_{i_{k}} \sum_{m=1}^{N} J_{j_{m}}}
$$

The total inductance of multiple-turn spiral inductors is the sum of the self-inductances of each turn and the mutual inductances between each turn and every other turn. In next step, we will describe how to compute $J$, the current distribution, in the loop.
According to the characteristics of ideal conductors, the value of magnetic field in the vertical direction on the surface of an ideal conductor is zero. Therefore, from $\nabla \times \bar{A}=\bar{B}$, the magnetic field in $z$ direction is:

$$
B_{z}=\frac{1}{\rho} \cdot \frac{\partial\left(\rho A_{\theta}\right)}{\partial \rho}-\frac{1}{\rho} \frac{\partial A_{\rho}}{\partial \theta}=0
$$

where:

$$
A_{\rho}=0
$$

and

$$
A_{\theta}=\oint_{L} G_{L}\left(\rho / \rho^{\prime}\right) \cdot J \cdot d l^{\prime}
$$

The Green's function for circular loop wire is given by [4]

$$
\begin{gathered}
G_{L}\left(\rho / \rho^{\prime}\right)=\widehat{\theta} \frac{\mu_{0}}{4 \pi \rho \sqrt{\left(\rho+\rho^{\prime}\right)^{2}+\left(z-z^{\prime}\right)^{2}}}\left\{2 \left[\rho^{2}+\rho^{\prime 2}\right.\right. \\
\left.+\left(z-z^{\prime}\right)^{2}\right] F\left(\frac{\pi}{2}, \eta\right)-\left[\left(\rho-\rho^{\prime}\right)^{2}+\left(z-z^{\prime}\right)^{2}\right] \\
\left.\Pi\left(\frac{\pi}{2},-\eta^{2}, \eta\right)-\left[\left(\rho-\rho^{\prime}\right)^{2}+\left(z-z^{\prime}\right)^{2}\right] E\left(\frac{\pi}{2}, \eta\right)\right\}
\end{gathered}
$$

where $F\left(\frac{\pi}{2}, \eta\right), E\left(\frac{\pi}{2}, \eta\right)$ and $\Pi\left(\frac{\pi}{2},-\eta^{2}, \eta\right)$ are the complete elliptic integral of the first, second and third kind with modulus $\eta$ respectively. The integral equation (8) can be solved by the Method of Moments. For an inductor of $N$ turns, each surface of conductor loop is subdivided into $M$ sub-loops of width $W / M$, and every sub-loop of $N$ turn is denoted as $1,2, \cdots, M ; M+1, M+2, \cdots, 2 M$; $\cdots, N M$, respectively. The current distribution on each sub-loop surface is assumed to be constant; in the other words, each concentric turn is assumed to carry the same current $I$ as the original spiral turn. We get

$$
\left[<A_{\theta n}, \varpi_{m}>\right]\left[\alpha_{n}\right]=\left[<\varpi_{m}, \frac{1}{\rho_{m}}>\right]
$$

where $\alpha_{i}$ is the coefficient of current densities to be determined in the sub-loops, and $\varpi_{m}$ the weight function.

Fig.5 shows the current distribution along the cross section of a three-turn inductor strip. The currents are pushed to their edge by the mutual coupling. After the current distribution is obtained, the inductance of the inductor can be calculated by the Eq.5 and Eq.1.

Using the new Green's Function, the computing time decrease significantly. One structre inductor of 3.5 turns is experimentlly examined. With the width of metal microstrip $w=5 \mu \mathrm{m}$, microstrip space $s=5 \mu \mathrm{m}$, the substrate permittivity $\varepsilon_{r}=12.9$, and substrate high $h=100 \mu m$, the results are seen in Fig.6. 


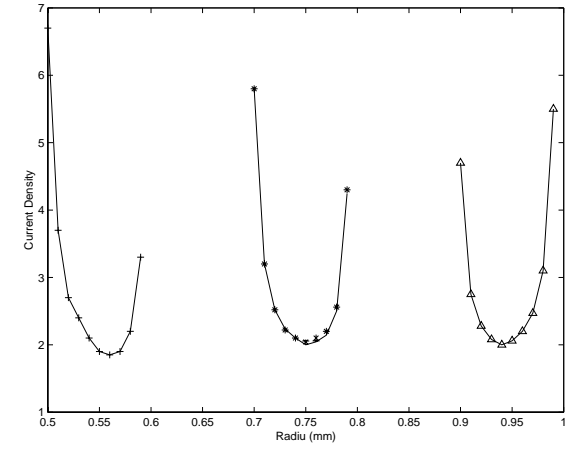

Fig. 5. Current distribution (Radiu $=1000 \mu m$, Turn=3)

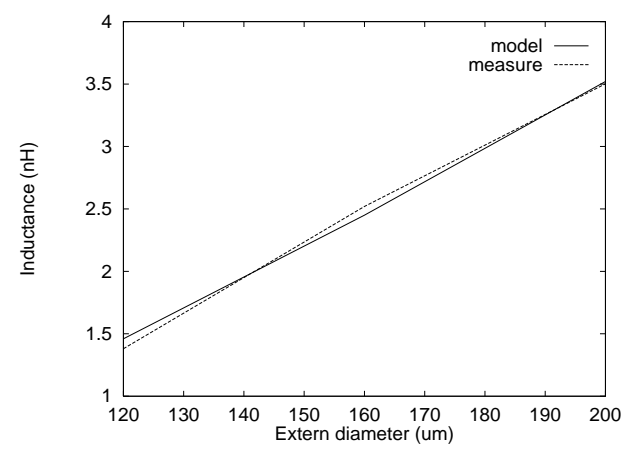

Fig. 6. Inductance as a function of extern diameter

\section{iII. Calculation of Capacitance}

The Green's Function of a circular wire loop with radius $\rho$ and a unit line charge density in a homogeneous dielectric with permittivity $\varepsilon$ can be calculated by

$$
G_{c}\left(\rho, z / \rho^{\prime}, z^{\prime}\right)=\frac{\rho^{\prime}}{\pi \varepsilon \sqrt{\left(\rho+\rho^{\prime}\right)^{2}+\left(z-z^{\prime}\right)^{2}}} F\left(\frac{\pi}{2}, \eta_{0}\right)
$$

where $F\left(\frac{\pi}{2}, \eta_{0}\right)$ is the complete elliptic integral function of the first kind and

$$
\eta_{0}=\sqrt{\frac{4 \rho \rho^{\prime}}{\left(\rho+\rho^{\prime}\right)^{2}+\left(z-z^{\prime}\right)^{2}}}
$$

But we can't write the Green's Function of the multi-layer substrates directly. In the next section we will describe how to solve this problem.

\section{A. Computing Green's Function for Multilayer Substrates Using Priorited Wave-tracing}

The image method is one of the most popular approaches for capacitance extract in layered media. But its application is usually limited to media with only a small number of layers for the following reasons:

- For more than two layers, the infinite image series expression is relatively hard to derive and implement;

- There is no robust convergence testing procedure for the image series. Moreover, in the far field, an enormous number of images are required to converge to a reasonable tolerance.

For multilayer media, the traditional image method is usually abandoned because of the above difficulties. Instead, an equivalent-source formulation is employed where the dielectric interfaces are discretized.

After carefully inspecting the physical meaning of the spectral Green's function, we found the wave-tracing algorithm very suitable to generalize the image method. At the initial time, the current source generates starting voltage waves (up and down) at the excitation point. The two waves can independently propagate. If the wave meets an impedance discontinuity, it splits into a reflected wave and a transmitted wave according to the boundary conditions. The reflected and transmitted waves then independently propagate. Each wave is attenuated by the splitting process and the distance it travels. Waves passing the observation point are called images. Fig.7 illustrates the wave propagation process.

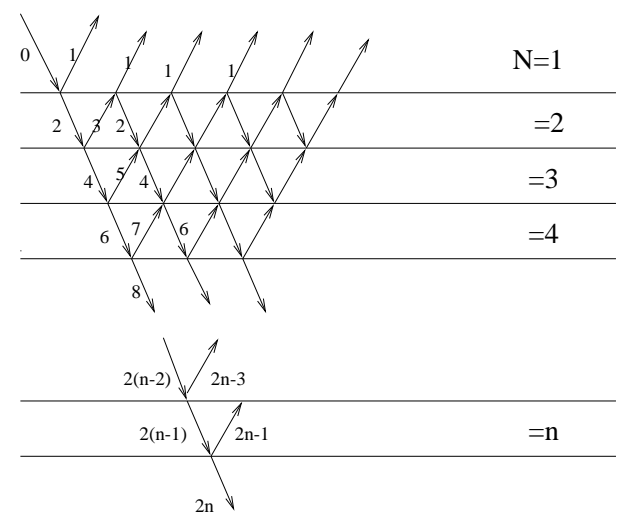

Fig. 7. The process of the wave propagation.

Wave-tracing is a general image tracing method for multilayered structure with arbitrary number of layers. However, in the real implementation, the brute-force version (TRACE), which is essentially a binary tree traversing, has two disadvantages[5]:

- Its blind tracing will quickly explode the procedure 
by exponentially increasing the generated waves, e.g., for level $n$, we have $2^{n}$ waves to trace;

- There is no robust convergence testing procedure.

The Prioritized Wave-tracing method was introduced in [5]. Rather than a naive implementation which recursively traces waves, each wave is instead assigned a key value which is calculated from the wave's amplitude and distance. Waves are dynamically sorted in a Priority Queue (PQ) with the strongest ones at the head. The key advantage of this implementation is that only the dominant waves are traced, and a good stopping criterion is available. Based on this algorithm, we derived the multiplayer substrate Green's Function

$$
G_{c}\left(\rho, z / \rho, z^{\prime}\right)=\sum_{i=0}^{N} G_{c, i}\left(\rho, h_{i} / \rho^{\prime}, z^{\prime}\right)
$$

where $G_{c, i}\left(\rho, h_{i} / \rho^{\prime}, z^{\prime}\right)$ is the $i^{t h}$ extracted image Green's Function from the head of Priority Queue after repetitious reflection and transmittion in the multi-layered substrates. In Eq.13,

$$
G_{c, i}\left(\rho, h_{i} / \rho^{\prime}, z^{\prime}\right)=\frac{m_{i \cdot} \rho^{\prime}}{\pi \varepsilon \sqrt{\left(\rho+\rho^{\prime}\right)^{2}+\left(h_{i}-z^{\prime}\right)^{2}}} F\left(\frac{\pi}{2}, \eta_{i}\right)
$$

where $m_{i}$ is the magnitude of the $i^{t h}$ image wave, $h_{i}$ the distance the $i^{t h}$ image wave propagates, and

$$
\eta_{i}=\sqrt{\frac{4 \rho \rho^{\prime}}{\left(\rho+\rho^{\prime}\right)^{2}+\left(h_{i}-z^{\prime}\right)^{2}}}
$$

For $N$ turns inductance, the total voltage potential at an arbitrary point $(\rho, z)$ on the $i^{\text {th }}$ turn

$$
\phi_{i}=\sum_{j=1}^{N} \int_{\rho_{j}}^{\rho_{j}+w} \sigma_{j}\left(\rho^{\prime}, z^{\prime}\right) \cdot G_{c}\left(\rho, z / \rho^{\prime}, z^{\prime}\right) \cdot d \rho^{\prime}
$$

where $\sigma_{j}\left(\rho^{\prime}, z^{\prime}\right)$ is the charge distribution functions on the $j^{\text {th }}$ turn.

\section{B. Charge Distribution}

When the point $P(\rho, z)$ is taken on the surface of the stripline of inductor, the voltage on the surface of the stripline can be obtained from Eq.16. Since every turn of the inductor is regarded as a perfect conductor, the tangential electric fields on its surface must be zero, the potential of every point on the surface of each turn must be equivalent. If the voltages on the surfaces of each turns $\phi_{i}$ $(i=1,2, \cdots, N)$ are given as $V_{1}, V_{2}, \cdots, V_{N}$, respectively, the charge distribution on each turn stripline surface can be solved by Eq.16. The integral equation can be easily solved by MoM. Thus, we have

$$
\left[<\varpi_{m}, L\left(\sigma_{n}\right)>\right]\left[\alpha_{n}\right]=\left[<\varpi_{m}, \phi>\right]
$$

where $\alpha_{i}$ is the coefficient of charge density to be determined in the sub-loops, and $\varpi_{m}$ the weight function. From the charge density distributions, the charges in each turns can be solved. The relationship of charge in each turns and the voltages can be expressed by

$$
\left[\begin{array}{c}
Q_{1} \\
Q_{2} \\
\vdots \\
Q_{N}
\end{array}\right]=\left[\begin{array}{cccc}
\beta_{11} & \beta_{12} & \cdots & \beta_{1 N} \\
\beta_{21} & \beta_{22} & \cdots & \beta_{2 N} \\
\vdots & \vdots & \vdots & \vdots \\
\beta_{N 1} & \beta_{N 2} & \cdots & \beta_{N N}
\end{array}\right]\left[\begin{array}{c}
V_{1} \\
V_{2} \\
\vdots \\
V_{N}
\end{array}\right]
$$

where $\left[\beta_{i j}\right]$ is the matrix of capacitance parameter. The self-capacitance of each turn is given by

$$
C_{j j}=\beta_{j 1}+\beta_{j 2}+\beta_{j 3}+\cdots+\beta_{j N}
$$

and the mutual capacitance between $i^{\text {th }}$ turn and $j^{\text {th }}$ turn. can be obtained by

$$
C_{i j}=-\beta_{i j}
$$

where $i \neq j$. When $i=j, C_{i j}$ is the capacitance of $j^{t h}$ turn to the ground.

\section{ExpERIMENTAL RESUlts}

We have designed and fabricated a suite of inductors with different structures. They are integrated in a threelayer substrates. The parameters of the three-layer substrates are: silicon $\left(\varepsilon=11.8, h_{1}=650 \mu m\right)$, polyimide $\left(\varepsilon=3.8, h_{2}=3.0 \mu \mathrm{m}\right)$, and a mask $\left(\varepsilon=3.0, h_{3}=10 \mu \mathrm{m}\right)$. Metalization sits on the second layer. This structure has slow decaying wave inside the polyimide and mask layers, and fast decaying in the silicon substrate layer.

Fig.8, Fig.9 and Fig.10 show good agreement between

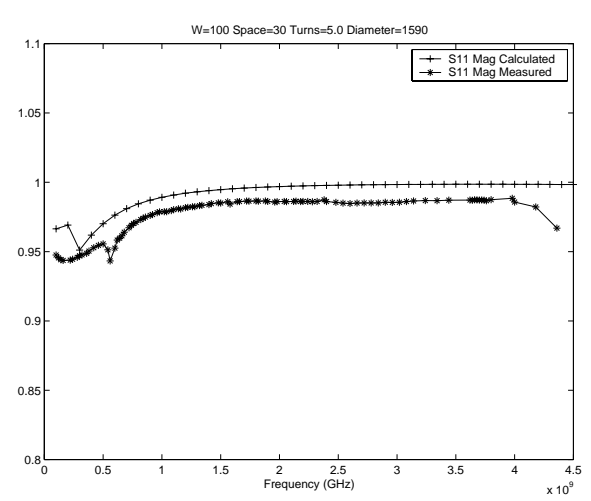

Fig. 8. Compared with measured results of magnitude of $S_{11}$ Parameter. Conductor width $=100 \mu \mathrm{m}$, space $=30 \mu \mathrm{m}$, turn $=5.0$ and external diameter $=1590 \mu \mathrm{m}$

$\mathbf{S}$ parameter computed by our method and that obtained from measurement, and Fig.11 shows the $\mathbf{Y}$ parameter 
produced by our method match well with that produced by the FMMS[6]. However, our method is two orders of magnitude faster than FMMS.

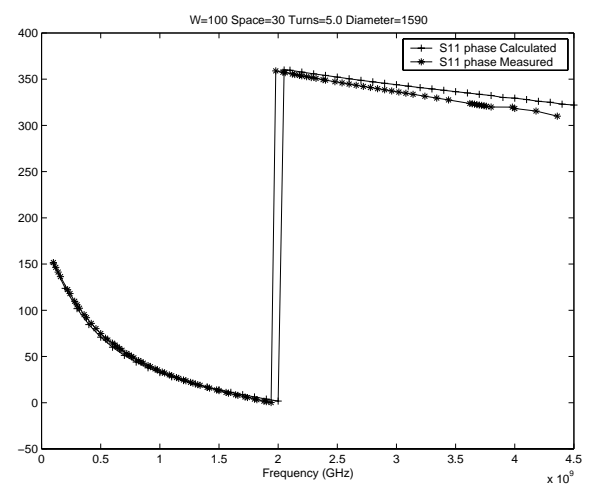

Fig. 9. Cpmpared with measured results of phase of $S_{11}$ Parameter. Conductor width $=100 \mu \mathrm{m}$, space $=30 \mu \mathrm{m}$, turn $=5.0$ and external diameter $=1590 \mu \mathrm{m}$

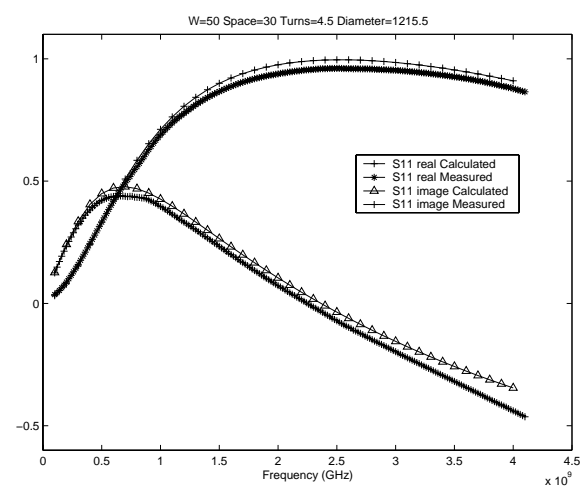

Fig. 10. Cpmpared with measured results of $S_{11}$ Parameter. Conductor width $=50 \mu \mathrm{m}$, space $=30 \mu \mathrm{m}$, turn $=4.5$ and external diameter $=1215.5 \mu \mathrm{m}$

In this paper we present a new subdomain partitioning technique for parameter extraction of integrated spiral inductors on SIP. This approach efficiently reduce the order of matrix from thousands (FMMS) to only hundreds and significantly speed up the parameter extraction. Experimental results demonstrate that the method is in good agreement with the measurement, and two orders of magnitude faster than FMMS.

\section{ACKnowledgements}

The authors would like to thank Dr. King L. Tai and Dr. Robort C. Frye for helpful discuss. The test suite of

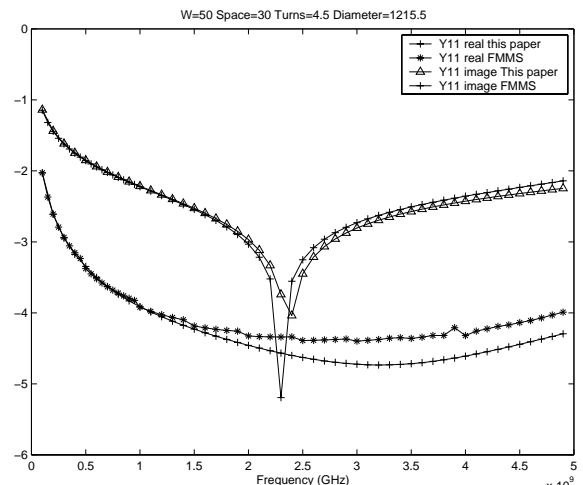

Fig. 11. Compared with FMMS in $Y_{11}$ Parameter. Conductor width $=50 \mu \mathrm{m}$, space $=30 \mu \mathrm{m}$, turn $=4.5$ and external diameter $=1215.5 \mu \mathrm{m}$.

the integrated inductor was fabricated by Bell laboratories, Lucent Technologies.

\section{REFERENCES}

[1] H. M. Greenhouse, "Design of planar rectangular microelectronic inductors," IEEE transactions on Parts, Hybrids, and Packaging, VOL, PHP-10 No.2, June 1974 PP.101-108.

[2] E. Frlan, "Computer aided design of square spiral transformers and inductors," IEEE MTT-S Digest, PP.661-664, 1989.

[3] E. Pettenpaul, "CAD models of lumped elements on GaAs up to 18GHz," IEEE Tran. On MTT-VOL.36, PP.294-303, 1988

[4] Minqing Liu, "Computer aided design of planar spiral inductor for MMIC," Chinese Journal of Radio Science, Proceedings of ICRS95. PP.389-393, 1995.

[5] Jinsong Zhao, "Numerical and experimental modeling of embedded passive elements for wireless applications,"

Doctors thesis Univ. of California at Santa Cruz, 1997.

[6] Sharad Kapur and Jinsong Zhao, "A fast method of moments solver for efficient parameter extraction of MCMs," DAC 97. CA, PP.141-146.

[7] L.Greengard and V. Rokhlin, "A fast algorithm for particle simulations" Journal of Computional Physics, 73(2):325-348, December 1987.

[8] S. Kapur, "High-order quadratures for singular functions with applications to Fourier and Hankel transforms," Ph.D thesis, Yale University Department of Computer Science, YALEU/DCS/RR-108 4, April 1995.

[9] K.Nabors, F.T.Korsmeyer, F.T.Leighton, J.White. "Preconditioned, adaptive, multipole-accelerated iterative methods for three-dimensional first-kind integral equations of potential theory," Sci. Comput., Vol 15(3):713-735, May 1994.

[10] J. Stoer and R. Bulirsch, "Introduction to Numerical Analysis," Springer-Verlag, NY,1979.

[11] Arun Balakrishnan, William D. Palmer, "Inductance of planar rectangular-spiral strip conductors for low-profile inductors," em IEEE MTT-S Digest, 1992. 